\title{
The effect of spatial frequency on global precedence and hemispheric differences
}

\author{
RONALD HÜBNER \\ Technische Universität Braunschweig, Braunschweig, Germany
}

\begin{abstract}
There are many conditions in which identification proceeds faster for the global form of a hierarchical pattern than for its local parts. Since the global form usually contains more lower spatial frequencies than do the local forms, it has frequently been suggested that the higher transmission rate of low spatial frequencies is responsible for the global advantage. There are also functional hemispheric differences. While the right hemisphere is better at processing global information, the left hemisphere has an advantage with respect to local information. In accordance with the spatial-frequency hypothesis, it has been speculated that this difference is due to a differential capacity of the hemispheres for processing low and high spatial frequencies. To test whether low spatial frequencies were responsible for the global advantage and/or for the observed hemispheric differences, two experiments were carried out with unfiltered and highpass-filtered compound-letter stimuli presented at the left, right, or center visual field. The first experiment, in which the target level was randomized in each trial block, revealed that low spatial frequencies were not necessary for either global advantage or for hemispheric differences. Highpass filtering merely increased the response times. In the second experiment, the target level was held constant in each block. This generally increased the speed of responding and produced interactions between filtering and global-local processing. It was concluded that both sensory and attentional or control mechanisms were responsible for global precedence and that the hemispheres differed with respect to the latter.
\end{abstract}

Since the pioneering paper of Navon (1977; also see Kinchla, 1974), the time course of the processing of hierarchical stimuli has been extensively investigated. Navon used so-called compound letters, which are global letter forms constructed from identical smaller local letters (see Figure 1). Usually, it is easy to focus one's attention on either the local or the global level. Interestingly, Navon found that the identification of the global form proceeded faster than that of the local letters. Moreover, if the information at the global and local levels was conflicting, then global information interfered with local processing, but not vice versa. I will denote a response time advantage for the processing of global forms together with an interference asymmetry as global advantage. To account for the global advantage observed in his data, Navon formulated the global-precedence hypothesis, which states that the information is processed serially at both levels and that the global information is processed first.

Meanwhile, it has been demonstrated that the global response time advantage varies over a wide range and can even become negative (e.g., Lamb \& Robertson, 1989). Thus, global advantage does not hold generally but depends on such various factors as visual angle (Kinchla \& Wolfe, 1979), the spacing of the local letters (Martin, 1979), retinal eccentricity (Pomerantz, 1983), and Gestalt

Thanks are extended to Dirk Vorberg, David Boles, and two anonymous reviewers for their very helpful comments on earlier versions of this manuscript. Address correspondence to R. Hübner, Technische Universität Braunschweig, Institut für Psychologie, Spielmannstr. 19, D-38106 Braunschweig, Germany (e-mail: r.huebner@tu-bs.de). qualities (LaGasse, 1993). Nevertheless, there are many conditions in which a global advantage occurs, and the question that arises is whether it is caused by sensory or attentional mechanisms (see Hoffman, 1980; Miller, 1981; Kinchla, Solis-Macias, \& Hoffman, 1983; Stoffer, 1993).

Potentially, at least two sensory mechanisms are relevant for the global advantage; both are related to the hypothesis that the human visual system acts as a spatial-frequency analyzer (see Campbell \& Robson, 1968; DeValois \& DeValois, 1988). In this conception, a stimulus is processed by a number of channels that are differentially sensitive to certain spatial frequencies. Attention to the local or global level of a compound stimulus could then be mediated by the selection or differential weighting of appropriate spatial-frequency channels (cf. Julesz \& Papathomas 1984; Hübner, 1993, 1996).

The first mechanism relevant for the global advantage is that while the sensitivity of the visual system varies across the spatial-frequency range, so does its transmission time. It is well known that low spatial frequencies are transmitted faster than high spatial frequencies (e.g., Breitmeyer, 1975; Lupp, Hauske, \& Wolf, 1976). Since the global letter forms of compound stimuli usually contain more low spatial frequencies than do the local letters, the transmission time difference is a possible candidate for explaining the global advantage. Such an account, for instance, is supported by the work of Sergent (1982), who demonstrated that with very brief exposure durations of the stimulus, the global form of a compound stimulus could still be accurately identified, whereas the local form could not. Since, at very brief exposure times, only low spatial 

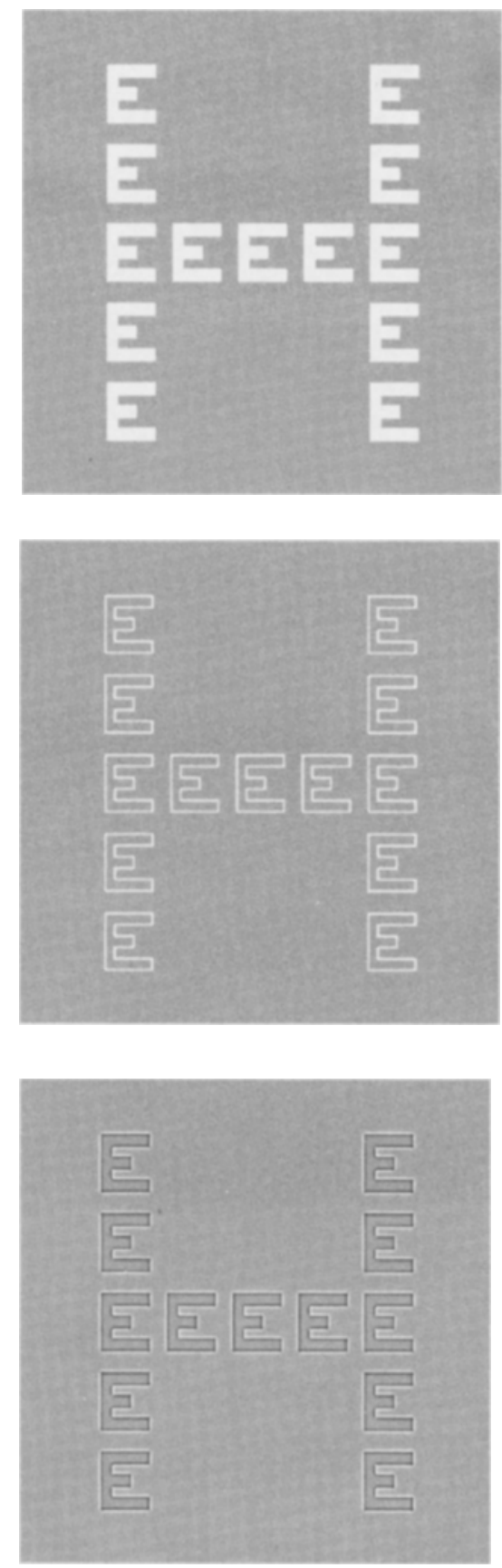

Figure 1. Examples of the stimuli used in the experiments. The upper compound letter is a filled, the middle an outlined, and the lower a highpass-filtered stimulus.

frequencies are available (Breitmeyer \& Ganz, 1977), they might generally be responsible for the global advantage. That the spatial-frequency content is important for the different perceptual characteristics of local and global forms has also been suggested by Shulman, Sullivan, Gish, and Sakoda (1986). They showed that the responses to global structures were selectively slowed by adaptation to low spatial frequencies, whereas the re- sponses to local structures were slowed mainly by adaptation to high spatial frequencies.

A more direct method for investigating the role of low spatial frequencies for global advantage is to use compound stimuli that do not contain low spatial frequencies. This method, for instance, was employed by Badcock, Whitworth, Badcock, and Lovegrove (1990). They presented highpass-filtered stimuli and found that the response time advantage for global forms vanished under this condition. A similar result was obtained by LaGasse (1993), who observed no global advantage with highpassfiltered stimuli and concluded that low spatial frequencies were necessary for global advantage. Another method for constructing compound stimuli without low spatial frequencies is to employ so-called contrast-balanced dots. With such stimuli, Hughes, Fendrich, and Reuter-Lorenz (1990) even found local-on-global interference.

A second sensory mechanism, potentially responsible for the global advantage, is related to asymmetric inhibition between spatial-frequency channels. The channels can be roughly classified as either transient or sustained. While transient channels respond mainly to low spatial frequencies, thereby signaling the stimulus on- or offset, the sustained channels respond steadily to medium and high spatial frequencies during the presence of a stimulus (e.g., Felipe, Buades, \& Artigas, 1993; Tolhurst, 1975). The important point with respect to global advantage is that the transient channels can inhibit the sustained channels but not vice versa (see Breitmeyer, 1984). For instance, Hughes (1986) presented compound stimuli consisting of two gratings with spatial frequencies of 0.5 and $5 \mathrm{cpd}$, respectively, and an orientation that was either horizontal or vertical. The subjects had to indicate the orientation of the target grating, where the nontarget grating could have either the target's orientation or an orthogonal one. It turned out that the response times for identifying the orientation of the high-spatial-frequency component were significantly slowed by an orthogonal low-spatialfrequency component, whereas the response times for the low-spatial-frequency components were not affected by an orthogonal high-spatial-frequency component. That transient activation was responsible for the asymmetric effect is supported by the additional result that the interference of low on high spatial frequency was reduced when the stimulus onsets were gradual rather than abrupt.

That inhibitory interactions between spatial-frequency channels are responsible for the global advantage has also been suggested by Lovegrove, Lehmkuhle, Baro, and Garzia (1991), who investigated the effect of uniform field flicker (UFF) on the processing of compound stimuli. UFF is known to affect response times to low but not to high spatial frequencies. It turned out that a UFF background increased the response times to global forms but decreased those to local forms. The latter result seems to indicate that the high-spatial-frequency channels are disinhibited when the transient channels are masked by the UFF. The asymmetric inhibition between sustained and transient channels could also explain con- 
ditions where a local response time advantage occurs together with global-on-local interference, that is, the faster process is affected by the slower one (e.g., Lamb \& Robertson, 1989). These results led to the hypothesis that the information at both levels was processed in parallel and that low spatial frequencies inhibited the processing of high spatial frequencies but not vice versa.

Together, these results suggest that low-level visual mechanisms are, at least partly, responsible for the globaladvantage effect. However, there is at least one negative finding. Recently, Lamb and Yund (1993) compared responses to ordinary, which they called "bright," and contrast-balanced compound letters. They found that the responses to the global structure were slowed for the latter stimuli as compared with the responses to the global structure of the bright stimuli, whereas the responses to the local letters and the substantial interference effects between the levels remained largely unaffected by the absence of low spatial frequencies.

As an alternative to these sensory mechanisms, it has been suggested that the global advantage is a postperceptual phenomenon attributable to attentional mechanisms (Boer \& Keuss, 1982; Kinchla et al., 1983; Miller, 1981; Ward, 1982). This approach usually assumes that both local and global sensory information is encoded in parallel and is available at the same time. Several results support this assumption. For instance, Miller (1981) employed a task in which the target could appear at the local and the global levels (divided attention). It turned out that the local information affected the responses even when the global information was sufficient for determining the response. Also, Boer and Keuss (1982), after analyzing speed-accuracy tradeoff functions, concluded that the availability of local and global aspects of a stimulus followed a similar time course in perception.

A possible attentional mechanism for explaining the global advantage, then, is to assume a spatial selection mechanism, such as the so-called zoomlens model (e.g., Eriksen \& St. James, 1986), and to postulate a selection preference for global information. The zoom-lens metaphor assumes an attentional spatial "spotlight" (see also Posner, 1980), which is variable in size and position within the visual field and in which stimuli are preferentially processed. To change the focus from a local to the global form, one has to increase the diameter of the spotlight.

In his attention-switching/-sharing model, Ward (1982) assumed such a zoomlens mechanism, which he called "attentional frame," and postulated two factors that determined the speed of information processing at a given level: (1) the attentional resources allocated to that level, and (2) the conspicuity or saliency of the features at that level. Interference between the levels is assumed to be a function of the imbalance in resource allocation and saliency between the levels. Ward derived his model from an experiment with a double-identification task, where the subjects had to respond to two compound stimuli in short succession. He found that if the level was identical for the first and second judgments, there was a considerable response time advantage over the condition in which the subjects had to switch attention. The advantage, which he called "level-readiness effect," occurred for targets at the local and global levels. Moreover, if the subjects had to respond to the local level of the first stimulus, then the global advantage was reversed for the second stimulus. Within this attentional conception, global advantage is explained by the assumption that, given no preferred resource allocation to one level, the global features, at least for stimuli of a certain size (cf. Kinchla \& Wolfe, 1979), are usually more salient than the local ones and, therefore, are processed preferentially.

However, the question that arises is: Why are the global forms more salient? An answer to this has been suggested by a recent approach, in which sensory and attentional mechanisms were combined to explain global advantage. By referring to Yantis and Jonides (1984), Stoffer (1993) speculated that transient activity, induced by abrupt visual onsets, captured attention or determined the diameter of the attentional spotlight in favor of global processing, and that this process might cause the greater saliency of global features. By employing the no-onset procedure of Todd and Van Gelder (1979), he was indeed able to prevent the global advantage. However, it has recently been observed that the appearance of objects can capture attention even when there is no luminance change, that is, no transient activation (Yantis \& Hillstrom, 1994). Generally, the questions that are still open are: Under what task conditions can which stimulus features capture an observer's attention? Do visual onsets play a special role? And how are the features related to stimulus saliency? (Cf. Jonides \& Yantis, 1988; Todd \& Kramer, 1994.)

The processing of global and local structures has also been related to the functional asymmetries between the hemispheres of the human brain. There is growing evidence that the right hemisphere (RH) and the left hemisphere (LH) are specialized for processing global and local structures, respectively (cf. Bradshaw \& Nettleton, 1981). Several attempts were made to test this hypothesis by employing compound-letter stimuli. Unfortunately, while some experiments found the expected differences between the hemispheres with respect to global-local processing (Martin, 1979; Robertson, Lamb, \& Zaidel, 1993; Sergent, 1982), others failed (Alivisatos \& Wilding, 1982; Boles, 1984; Van Kleeck, 1989). A meta-analysis by Van Kleeck revealed that even in experiments where the differences were not significant, the results point in the predicted direction. However, Boles and Karner (1996) recently even found effects in the opposite direction. Thus, the experimental results remain inconclusive. However, the hypothesis that there are functional hemispheric differences with respect to the processing of global and local information is strongly supported by neuropsychological results (for an overview, see Robertson \& Lamb, 1991). For instance, it has been demonstrated that brain lesions that prevent communication between the hemispheres eliminate the interference effects (cf. Humphreys, Riddoch, \& Quinlan, 1985; Robertson et al., 1993).

Interestingly, the hemispheres have also been associated with specific capabilities of processing spatial fre- 
quencies (e.g., Sergent, 1982). However, the difference between the hemispheres does not concern the extraction of spatial frequencies, but rather the utilization of the information provided by different spatial-frequency channels. It has been proposed that the RH has a greater capacity for performing cognitive operations on the basis of low spatial frequencies, whereas the $\mathrm{LH}$ is better equipped for operating on the output of high-spatial-frequency channels (Sergent, 1987). This hypothesis implies that there should be hemispheric differences only for higher cognitive processes in complex tasks and not for such simple tasks as detection. To test this prediction, Peterzell, Harvey, and Hardyck (1989) varied stimulus and task factors. First, they measured contrast sensitivity and visual persistence for sinusoidal gratings presented at the left visual field (LVF) and at the right visual field (RVF). As expected, they found no differences. Then, in a further experiment, they employed a letter-identification task which they thought was sufficiently complex for inducing hemispheric differences. They varied the spatial-frequency content of the stimuli by changing their size and by bandpass filtering. As a result, for this task too, except for a response bias, no differences between the hemispheres were observed. The response bias, however, did not correspond to a certain spatial-frequency content of the stimuli. Rather, it was associated with the reduction of stimulus quality by bandpass filtering. Their results led Peterzell et al. (1989) to agree with the hypothesis that the hemispheres are functionally equivalent at early stages of processing, and that asymmetries emerge only at later cognitive stages. However, they do not share the idea that the hemispheres are specialized for processing specific spatial-frequency contents.

It is worth mentioning that, even for their complex task, Peterzell et al. (1989) did not employ compound stimuli, which constitutes an important difference from experiments designed for investigating global-local processing. Consequently, their subjects did not have to ignore information on a different level or spatial scale. That this difference might be crucial is suggested by the results of Kitterle, Christman, and Conesa (1993), who used compound gratings to investigate hemispheric differences. They found that the magnitude and direction of interference between spatial-frequency channels depended on the visual field of presentation. For stimuli presented at the LVF, there were large interference effects of low-onhigh spatial-frequency channels but no interference in the other direction. For stimuli presented at the RVF, there was interference in both directions. Thus, while Kitterle et al. (1993), contrary to Peterzell et al. (1989), found hemispheric differences with respect to spatial frequencies, they observed no response bias with respect to the visual fields. They suggest that the hemispheres differ in their modulation of interchannel inhibition. However, they mention that differences with respect to attentional processes might play a role. The latter view is also shared by Robertson and Lamb (1991). They argue that hemispheric differences with respect to spatial frequencies can be ob- served only for tasks that involve such higher order processes as discrimination.

As this short overview shows, despite the considerable number of experiments and ideas concerned with global advantage, its exact mechanisms are still not well understood. Generally, the question of whether global advantage has a sensory or attentional basis, or depends on both, is open. The results of studies that have investigated the contribution of low spatial frequencies are inconclusive. Also, the differences between the hemispheres with respect to their capabilities of processing low and high spatial frequencies or global and local information are not very clear.

The experiments that will be reported here examined the role of the spatial-frequency content of the stimuli for global precedence and possible hemispheric differences simultaneously. This should provide insight into the relations between the processing of global versus local features, the processing of high versus low spatial frequencies, and possible differential preferences of the hemispheres for any of these processes. Kitterle et al. (1993) have already used compound stimuli to investigate the interaction between the processing of different spatial frequencies and their modulation within each hemisphere. However, since they employed compound gratings, it is an open question as to whether their results are also valid for hierarchical stimuli and global versus local feature processing. This would be the case only if the spatialfrequency account of the global advantage was true.

In the present experiments, two types of unfiltered and one type of highpass-filtered compound-letter stimuli (see Figure 1) were presented at the center visual field and both visual hemifields. These stimuli allow for independent variation of global and local processing and of the spatial-frequency content. Two different types of unfiltered stimuli were chosen to also examine the effects of a spatial-frequency variation that is different from filtering. The two unfiltered stimulus types will be labeled as "filled" and "outlined," respectively (see Figure 1). The outlined stimuli have less energy at the low but more at the high spatial frequencies than do the filled stimuli. A more detailed spectral analysis of all three stimulus types will be given in the Method section of Experiment 1 .

The hypothesis was that global precedence depended strongly on the presence of low spatial frequencies. Thus, the global advantage should be reduced for the outlined as compared with the filled stimuli and be absent for the filtered stimuli. Furthermore, the RH was expected to process global information more effectively and local information less effectively than the LH. Consequently, an interaction between visual field and target level was expected.

\section{EXPERIMENT 1}

\section{Method}

\section{Stimuli}

Four different letters, "E," "F," "H," and "T" were used and mapped to two response categories $\{$ "E," "T"\}, and \{ $F$," "H"\}. Sixteen compound stimuli were created by combining all letters, where 
global letters were constructed from identical local letters in a $5 \times$ 5 grid. At a viewing distance of $127 \mathrm{~cm}$, the global letters extended a visual angle of $1.93^{\circ}$ horizontally and $2.34^{\circ}$ vertically, and the local letters extended $0.30^{\circ} \times 0.36^{\circ}$, respectively.

Three stimulus types were used: filled letters, outlined letters, and highpass-filtered letters. Examples of all three types are depicted in Figure 1. Highpass-filtered stimuli were produced by weighting the spectrum of the filled letters with that of the highpass version of a Gaussian filter (i.e., 1 minus the response function of a standard lowpass Gaussian filter) with a standard deviation of $8.20 \mathrm{cpd}$, and by transforming the result back to the spatial domain. The contrast (Michelson contrast) of the unfiltered letters was $52 \%$ and that of the filtered letters was $99 \%$. Background luminance was set to $41 \mathrm{~cd} / \mathrm{m}^{2}$.

To get an impression of the spectral differences between the stimulus types, the amplitude spectrum of each of the stimuli in Figure 1 was computed. The value of each point in the two-dimensional spectrum represents the amplitude of a certain spatial-frequency component with a certain orientation. Since the stimuli are constructed exclusively of horizontal and vertical lines, most of the stimulus energy is concentrated at these orientations. Therefore, for demonstrating amplitude differences, it is sufficient to consider only a one-dimensional part of the spectrum corresponding to one of these orientations.

The amplitude differences (in decibels) of the spatial-frequency components with a vertical orientation are shown in Figure 2. The dashed line represents the difference between the filled and the highpassed stimuli. As one can see, there is an amplitude difference of more than $70 \mathrm{~dB}$ at the very low spatial frequencies. With increas- ing spatial frequency, the difference decreases. The graph nicely illustrates the effect of the Gaussian highpass filter. The solid line represents the amplitude differences between the filled and the outlined stimuli. Obviously, the outlined stimuli also have lower amplitudes at low spatial frequencies. The difference is about $10 \mathrm{~dB}$ at the very low spatial frequencies and even higher for certain frequency bands. Moreover, the amplitude differences at the medium and high spatial frequencies are negative up to an amount of about $-15 \mathrm{~dB}$. Thus, unlike the filtered stimuli, which have only their low spatial frequencies attenuated, as compared with the filled stimuli, the outlined stimuli possess additional energy at medium and high spatial frequencies.

\section{Apparatus}

The stimuli were presented on a 19-in. color monitor (Miro, Type GDM-1965) with a resolution of $1,280 \times 1,024$ pixels, which was connected to a graphics board (Miro-Tiger) with 256 gray levels and a refresh rate of $75 \mathrm{~Hz}$ (noninterlaced). A personal computer $(\mathrm{PC})$ controlled stimuli presentation and response registration. The space average luminance for each gray level was measured with an L-1000 photometer from LMT Lichtmesstechnik, Berlin, and the data were used to create a gamma look-up table to relate the required luminances to the corresponding gray levels.

\section{Procedure}

The task of the subjects was to classify the letters by pressing one of two response buttons with the index or middle finger, respec-

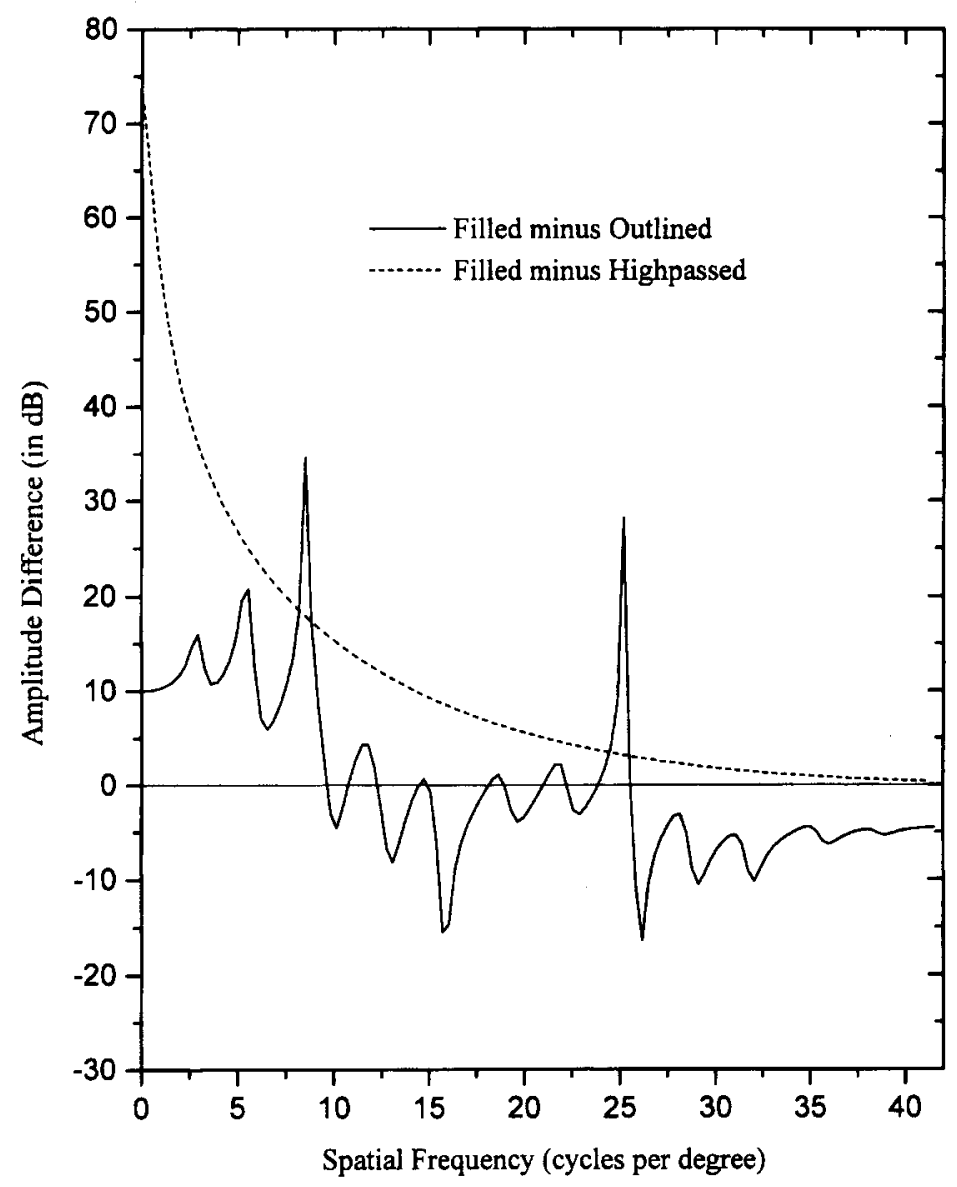

Figure 2. The amplitude-spectrum differences of the three stimuli in Figure 1. Only the values of the spatial-frequency components corresponding to the vertical orientation are considered. 
tively, of the same hand. Gender, mapping of the letter pairs to the response keys (or fingers), and responding hand were counterbalanced across subjects. For instance, some of the subjects who responded with the right hand, were to indicate the presence of " $E$ " or " $T$ " with the index finger and the presence of " $H$ " and " $F$ " with the middle finger. For the other subjects, the finger mapping was reversed.

Each trial started with the appearance of a fixation cross and a cue, both of which were centered on the screen. The cue was the outline of a rectangle, in a size that corresponded to that of the relevant letter level. After the subjects had started the trial by pressing a key with the hand that was not used for responding to the stimuli, a blank screen appeared for a random interval of $200-600 \mathrm{msec}$. This was followed by the stimulus, which was exposed for $196 \mathrm{msec}$ at one of three positions: left visual field (LVF), center visual field (CVF), or right visual field (RVF). Each of the stimuli presented at the RVF or LVF was positioned in such a way that its left or right border was aligned with the center of the screen. This relatively small eccentricity was chosen to keep the highpass filtered stimuli visible, since sensitivity decreases faster with eccentricity for the higher spatial frequencies than for the lower ones (see Graham, 1989).

All conditions were randomized, and errors were signaled by a tone. Altogether, there were 36 conditions: 3 stimulus positions $\times 3$ stimulus types $\times 2$ target levels (global, local) $\times 2$ consistency conditions (consistent, inconsistent). Since 8 different compound letters appeared in each condition, there were, altogether, 288 stimulus situations, which were split into 3 different blocks with five warm-up trials added to the beginning of each block. After some practice blocks, the 3 blocks were run 10 times, distributed over about 5 sessions. Altogether we obtained 80 responses for each condition per subject (altogether 2,880 trials for each subject).

\section{Subjects}

Eight right-handed (by self-report) subjects ( 4 male and 4 female), who ranged in age from 20 to 37 years and had normal or corrected-to-normal visual acuity, participated in the experiment.

\section{Results}

\section{Latencies}

To reduce the effect of extreme values, the latencies of each subject's correct responses for each condition were trimmed by disregarding the three slowest and the two fastest responses of each condition. An asymmetric trimming procedure was chosen because the obtained latency distributions were positively skewed. The means of the trimmed data were then subjected to a four-factor analysis of variance (ANOVA) for repeated measurements on all four factors - that is, position (LVF, CVF, RVF), stimulus type (filled, outlined, filtered), target level (global, local), and consistency (consistent, inconsistent).

The position factor turned out to be significant $[F(2,14)$ $=11.8, p<.001]$; this was due to the fact that the responses to the stimuli presented at the CVF were faster than those presented to the other visual fields (LVF, $659 \mathrm{msec}$; CVF, $638 \mathrm{msec}$; RVF, $655 \mathrm{msec}$ ). Furthermore, the stimulustype effect was significant $[F(2,14)=139, p<.001]$. Highpass filtering led to considerably slowed responses (695 $\mathrm{msec})$, compared with those for the filled $(625 \mathrm{msec})$ and outlined (632 msec) stimuli. However, even the relatively small difference between the latencies for the filled and outlined stimuli was significant $[F(1,7)=6.3$, $p<.05$ ]. Also significant were the target-level [global level, $636 \mathrm{msec}$, and local level, $664 \mathrm{msec} ; F(1,7)=$
$8.01, p<.05$ ], and consistency factors [consistent, $632 \mathrm{msec}$, and inconsistent, $668 \mathrm{msec} ; F(1,7)=20.8$, $p<.01]$.

As for target level, there was also a significant twoway interaction with position $[F(2,14)=19.3, p<.001]$. To test the hypothesis that the hemispheres were differentially sensitive to information at the different levels, the responses to the global as well as those to the local levels were compared for stimuli presented at the LVF and RVF. It turned out that global processing was significantly faster for stimuli presented at the LVF [627 vs. $648 \mathrm{msec}$; $F(1,7)=14.6, p<.01]$, while the processing of local information was faster for stimuli presented at the RVF $[658$ vs. $690 \mathrm{msec} ; F(1,7)=14.1, p<.01]$.

The ANOVA also showed a significant three-way interaction between position, target level, and consistency $[F(2,14)=8.59, p<.01]$. However, since there was also a significant four-way interaction between all factors $[F(4,28)=4.30, p<.01]$, the data were further analyzed by considering the results for the individual stimulus types separately. The mean latencies for the different conditions are depicted in Figure 3. As can be seen, there is considerable variability across the different conditions. For allowing possible interactions involving the position factor to be directly interpretable with respect to the processing differences between the hemispheres, only the data corresponding to the LVF and RVF were included in the subsequent analysis.

Filled stimuli. For this stimulus type, the target-level factor was significant $[F(1,7)=9.71, p<.05]$. Responses were faster to the global level $(613 \mathrm{msec})$ than those to the local level $(647 \mathrm{msec})$. Moreover, there was a significant two-way interaction between position and target level $[F(1,7)=49.9, p<.001]$, which mirrors that in the accumulated data. While the responses to the global level were faster for stimuli presented at the LVF $(604 \mathrm{msec})$ than for those presented at the RVF [623 msec; $F(1,7)=$ $27.3, p<.01]$, the opposite held true for the local level. Here, the responses were faster to the stimuli presented at the RVF $(634 \mathrm{msec})$ than those to stimuli presented at the LVF [660 msec; $F(1,7)=39.3, p<.001]$.

There was also a significant consistency effect $[F(1,7)=$ $16.8, p<.01]$. Responses to consistent stimuli were $35 \mathrm{msec}$ faster than those to inconsistent stimuli. This pattern of results indicates a global response time advantage for the filled stimuli.

Outlined stimuli. For the outlined stimuli, there is a significant consistency effect as well $[F(1,7)=20.3, p<$ $.01]$. Responses to consistent stimuli were $41 \mathrm{msec}$ faster than those to inconsistent stimuli. There was also a significant target-level effect $[F(1,7)=20.4, p<.01]$. Responses to the global level were $38 \mathrm{msec}$ faster than responses to the local level. Moreover, the position $\times$ target level interaction was significant $[F(1,7)=26.9, p<.01]$. As for the filled stimuli, responses to the global level were faster for LVF stimuli $(607 \mathrm{msec})$ than for RVF stimuli [626 msec; $F(1,7)=18.0, p<.01]$. On the other hand, responses to the local level were faster for RVF stimuli 


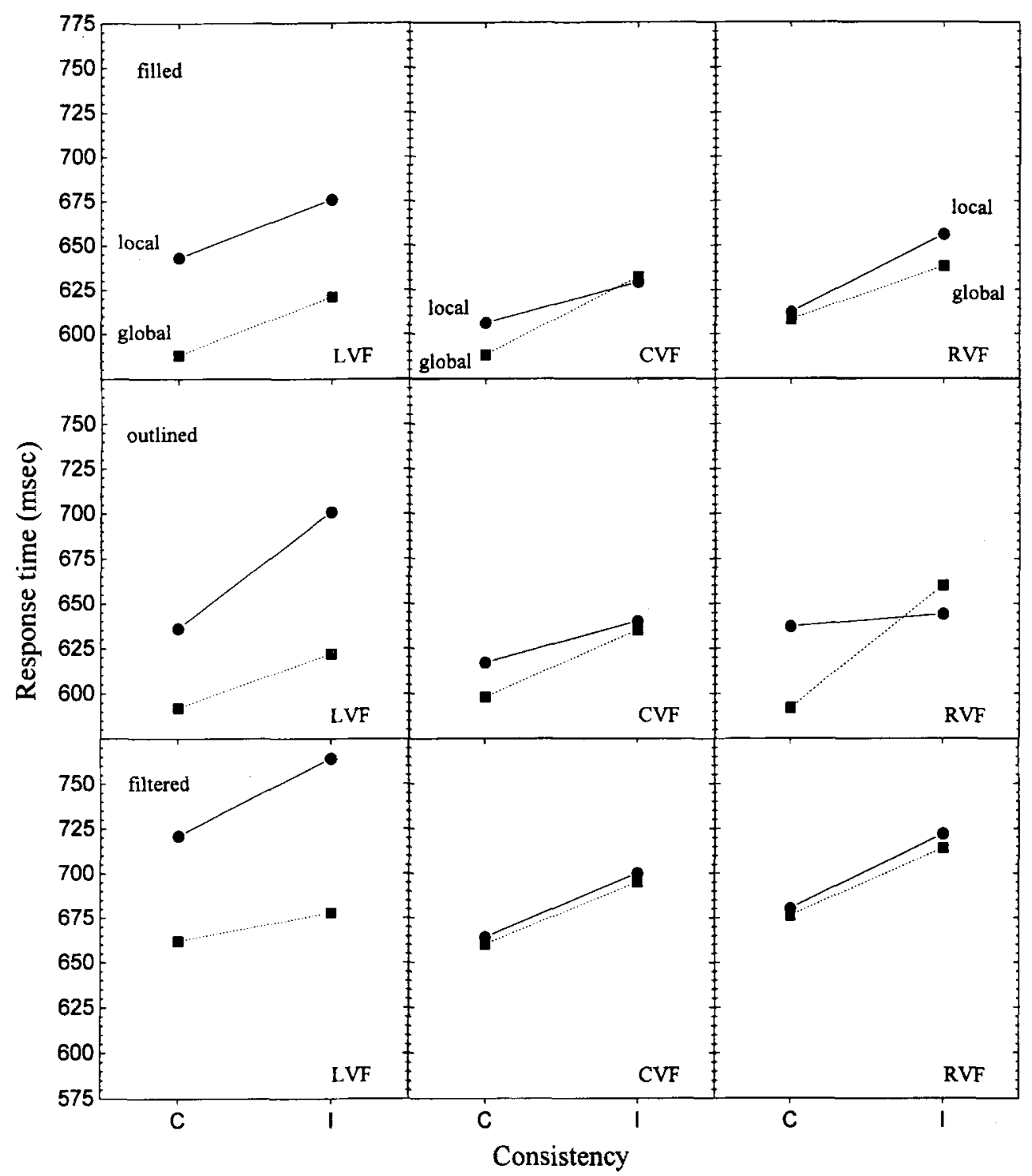

Figure 3. Results of Experiment 1.

$(640 \mathrm{msec})$ than for LVF stimuli $[669 \mathrm{msec} ; F(1,7)=$ $17.7, p<.01]$.

However, there was also a significant three-way interaction between position, level, and consistency $[F(1,7)=$ $21.8, p<.01]$. By inspecting Figure 3, it is obvious that the interaction between target level and consistency is quite different for the individual hemifields. The LVF data show a significant target-level effect $[F(1,7)=78.8, p<$ $.001]$, a significant consistency effect $[F(1,7)=33.8$, $p<.001]$, and a significant interaction $[F(1,7)=7.45, p<$ $.05]$. The consistency effect is larger for the local level $(65 \mathrm{msec})$ than for the global level $(30 \mathrm{msec})$.

The stimuli presented at the RVF also produced a consistency effect $[F(1,7)=10.4, p<.05]$ and a significant interaction $[F(1,7)=9.69, p<.05]$. Here, however, the consistency effect was larger for the global level $(68 \mathrm{msec})$ than for the local level $(7 \mathrm{msec})$.
Filtered stimuli. The filtered stimuli also produced a significant target-level effect $[F(1,7)=17.29, p<.01]$. Responses to the global level were $40 \mathrm{msec}$ faster than those to the local level. Moreover, there was a two-way interaction between position and target level $[F(1,7)=$ $11.9, p<.05]$. While the responses to the local level were faster for stimuli presented at the RVF [701 vs. $742 \mathrm{msec}$; $F(1,7)=5.98, p<.05]$, the latency difference for the global level was in the opposite direction (LVF, $670 \mathrm{msec}$; RVF, $695 \mathrm{msec})$, but failed to reach significance $[F(1,7)=$ $3.98, p<.08]$.

The filtered stimuli also produced a significant consistency effect $[F(1,7)=10.1, p<.05]$. Responses to consistent stimuli were 34 msec faster than those to inconsistent stimuli. Thus, the filtered stimuli produced a response time pattern that was rather similar to that for the filled stimuli. 
Error rates. Errors (see Table 1) occurred, on average, in only $4.6 \%$ of the trials. After an arcsin transformation of the error rates, they were subjected to a four-factor ANOVA for repeated measures on all factors. The result indicated a significant stimulus-type effect $[F(2,14)=$ $9.86, p<.01]$. More errors were made for the filtered stimuli (filled, $4.09 \%$; outlined, $4.07 \%$; filtered, $5.67 \%$ ). Also, the consistency effect was significant [consistent, $3.07 \%$; inconsistent, $6.14 \% ; F(1,7)=85.6, p<.001$ ] Furthermore, there was a significant position $\times$ target level interaction $[F(2,14)=3.85, p<.05]$, which is similar to that in the latency data.

Another significant two-way interaction was that between stimulus type and consistency $[F(2,14)=4.30, p<$ $.05]$. The error difference between the consistent and inconsistent conditions was less for the filtered than for the other stimuli. This was due to the fact that the filtered stimuli also produced relatively high error rates in the consistent conditions. Finally, there was a significant threeway interaction between position, target level, and consistency $[F(2,14)=8.58, p<.01]$. This was due to the relatively high error rate $(11 \%)$ for responses at the local level for inconsistent stimuli presented at the LVF; the other error rates lay between $2 \%$ and $5 \%$.

The analysis shows that the error rates point roughly in the same direction as the response times. Thus, tradeoff effects can be excluded.

\section{Discussion}

The data show that the responses to the stimuli presented at the CVF were faster than those to the stimuli presented at the LVF and RVF. Although the CVF data were not analyzed separately, it is obvious that there was no global advantage, which is in line with other results (e.g., Lamb \& Robertson, 1988). Even for the LVF and RVF stimuli there is no global advantage. However, there is an appreciable global response time advantage. Moreover, there are also striking hemispheric differences. The global response time advantage is more pronounced for the stimuli presented at the LVF. Furthermore, the differences are in the expected direction. The global level was processed faster for stimuli presented at the LVF, while the local level was processed faster for the RVF stimuli.

The large hemispheric differences are surprising considering the relatively large number of unsuccessful experiments. One reviewer raised the question of whether

Table 1

Error Rates of Experiment 1 in Percentages

\begin{tabular}{|c|c|c|c|c|c|c|c|}
\hline \multirow[b]{2}{*}{ Stimulus } & \multirow{2}{*}{$\begin{array}{l}\text { Target } \\
\text { Level }\end{array}$} & \multicolumn{2}{|c|}{ LVF } & \multicolumn{2}{|c|}{ CVF } & \multicolumn{2}{|c|}{ RVF } \\
\hline & & Con. & Incon. & Con. & Incon. & Con. & Incon \\
\hline \multirow[t]{2}{*}{ Filled } & global & 2.3 & 3.6 & 1.6 & 6.0 & 1.8 & 5.7 \\
\hline & local & 2.2 & 8.7 & 4.2 & 5.8 & 2.2 & 4.5 \\
\hline \multirow[t]{2}{*}{ Outlined } & global & 1.7 & 5.5 & 2.2 & 4.7 & 1.3 & 4.5 \\
\hline & local & 3.2 & 9.1 & 2.6 & 5.0 & 3.7 & 5.0 \\
\hline \multirow[t]{2}{*}{ Filtered } & global & 4.0 & 4.5 & 3.2 & 5.0 & 4.0 & 6.5 \\
\hline & local & 5.2 & 13.7 & 5.0 & 6.3 & 4.2 & 6.1 \\
\hline
\end{tabular}

Note - LVF $=$ left visual field, CVF = center visual field, RVF $=$ right visual field. Con. $=$ consistent, Incon. $=$ inconsistent. the large effect could be due to the asymmetric stimuli employed ("F" and "E"). Since the information for discriminating these stimuli differs on their left and right borders, it could be easier to identify these letters, at least in their global version, when they are presented at the LVF. Moreover, the number of local letters near the fixation point is different for LVF and RVF presentations. To test this possibility, the responses to the symmetric ("T" and "H") global stimuli were analyzed separately. The results were similar to that obtained with all the data. Most importantly, for these data, too, a significant position $\times$ target level interaction occurred $[F(2,14)=$ $12.4, p<.001]$. The differences are in the same direction as those in the main analysis.

That even small construction differences between the stimuli can cause large effects is demonstrated by the outlined stimuli. They produced extreme local-on-global interference for the RVF condition. However, local-onglobal interference is obvious in other conditions as well, and has been observed before (e.g., Boer \& Keuss, 1982; Lamb \& Robertson, 1988; Miller, 1981). It is also worth mentioning that Kitterle et al. (1993) found interference from high to low spatial frequency for stimuli presented at the RVF. One might be tempted to speculate that the increased energy at the high spatial frequencies could have produced the extreme local-on-global interference effect. However, since the reduction of low spatial frequencies by way of filtering had almost no effect on interference, such an explanation is questionable. Also, one should consider the effect as preliminary, as long as no other data demonstrate it to be valid.

Contrary to the hypothesis, highpass filtering did not eliminate the global response time advantage. If one compares the data for filled stimuli with the data for the filtered stimuli, then it is surprising that the filtering effect is so homogeneous across the different conditions. The results provide no support for the hypothesis that low spatial frequencies are responsible for the global advantage, and are thus in line with those of Lamb and Yund (1993). Rather, they indicate that the global advantage can be accounted for by attentional processes, which operate differently in both hemispheres. Thus, low spatial frequencies might be sufficient, but are not necessary, to produce global advantage.

In consideration of the other more successful filtering experiments, the question that arises is why filtering did not affect the global response time advantage here. One difference between experiments in which the removal of low spatial frequencies reduced the global advantage (e.g., Badcock et al., 1990; Hughes et al., 1990; LaGasse, 1993) and the one reported here is that, in the present experiment, the target level was randomized. ${ }^{I}$ The frequent switching between global and local processing could have prevented the subjects from optimally focusing their attention on a certain level. Similarly, in Lamb and Yund's (1993) study, where a global advantage occurred without low spatial frequencies, the subjects could not focus their attention entirely on one level because the target letter could appear at both levels. 
Important for this target-level switching might be task set, the configuration of the supervisory attentional system for the execution of the intended action. It has been shown that dynamically shifting between cognitive operations or task-relevant stimulus dimensions, which requires a reconfiguration of the cognitive system, can produce large costs in response times and error rates (e.g., Allport, Styles, \& Hsieh, 1994). Moreover, task-irrelevant stimulus attributes, which are linked via stimulus-response mappings to the task-relevant responses, can lead to a considerable cross-talk between the tasks (Rogers \& Monsell, 1995), and the cross-talk is increased, if a task switch is required, as compared with trials without a task switch.

Interestingly, a large percentage of switch costs has been found to be independent of the response-stimulus interval (Allport et al., 1994; Rogers \& Monsell, 1995). This fact resembles Ward's (1982) results, since he found a level-readiness effect not only within but also between trials, even with intertrial intervals of $2.5-3.5 \mathrm{sec}$. Thus, the level-readiness effects and the task-switch effects might depend on the same mechanisms.

In any case, one could speculate that an increased processing duration, due to a suboptimal task set or level focusing, might have reduced the sensory effects of the low spatial frequencies and increased the contribution of high spatial frequencies. Also, attentional or other higher order processes might have gained a relatively large weight during extended processing in the present experiment. If focusing to a level was indeed suboptimal, then one should find response time costs for switching from local to global, and vice versa, even though the subjects could start each trial by themselves without external time pressure. To test this hypothesis, the data were analyzed for sequential dependencies.

If there are task-switch costs, then one would expect that a correct response to a certain level would be faster after a correct response had been made to the same level in the preceding trial than it would be after a correct response had been made to the other level in the preceding trial. Thus we have four conditions: local after local (LL), local after global (GL), global after global (GG), and global after local (LG). A two-factor (target level $\times$ target level in the preceding trial) ANOVA with these data reveals a significant interaction $[F(1,7)=10.0, p<.05]$. Responses were faster when the response in the preceding trial was made to the same level. This holds for responses to the local level (GL, $695 \mathrm{msec} v s$. LL, $667 \mathrm{msec}$ ) as well as for responses to the global level (LG, $664 \mathrm{msec}$, vs. $\mathrm{GG}, 643 \mathrm{msec}$ ).

Thus, switching between the tasks or target levels produced response time costs. ${ }^{2}$ This supports the hypothesis that the task set was suboptimal in the present experiment, at least in some of the trials. It seems that, for an efficient processing of local or global information, the subjects have to configure their cognitive system appropriately, presumably by allocating attentional resources to the taskrelevant mechanisms. Such a task set remains valid for some period of time even after the stimulus has been re- moved, and can be switched, though only to a certain extent, to another level by voluntary or endogenous control (Rogers \& Monsell, 1995).

Allport et al. (1994) assume that the task-switching costs are due to task-set inertia, and that the reconfiguration of a cognitive system is a gradual process which improves over several trials. Thus, under conditions with frequent task switching, an optimal performance is hardly ever reached. However, Rogers and Monsell (1995), by employing an alternating runs paradigm, found that, at least for classifying numbers and characters, performance improves only between the first and second trial of a run and then remains constant. To see whether the sequential effects continue over more than two trials in the present experiment, responses, preceded by a sequence of correct responses to a certain level, were examined separately for sequence lengths of up to 4 . They were analyzed in such a way that the data corresponding to a certain sequence length were not included in the analysis of the data corresponding to any other examined sequence length. For instance, responses to the local level that were considered were preceded by sequences of exactly $1(\mathrm{GL})$, exactly 2 (GGL), exactly 3 (GGGL), or 4 or more (GGGGL) responses to the global level.

It turned out that the costs for switching to the global level, that is, the latency differences between responses to the global level preceded by responses to the same level and responses to the global level preceded by responses to the local level, increased slightly with sequence length $(20,13,32$, and $34 \mathrm{msec})$. This increase is more pronounced for switches to the local level $(20,20,43$, and $43 \mathrm{msec}$ ). Taken together, the increase in switch cost with sequence length is reliable $[r=.807, t(7)=3.35$, $p<.05]$. Thus, although the sequential effects are rather small, they support the task-set inertia assumption of Allport et al. (1994).

In any case, an experiment without task switching should reduce the response times. Therefore, the experiment was repeated with a constant target level in each trial block. This should allow the subjects to focus their attention more efficiently. It was expected that under this condition the attentional effects would be reduced and the sensory effects would be increased. Specifically, it was predicted that, for the filtered stimuli, the global response time advantage would be reduced as compared with the other stimuli. In addition, it was expected that the interference effects would decrease.

\section{EXPERIMENT 2}

\section{Method}

The method was identical to that in Experiment 1, except that the target level (local and global) was constant in each trial block. Since half of the 288 different stimulus conditions corresponded to the local target level and the other half to the global target level, the 144 conditions for each level were split into two blocks of 72 . After some practice trials, half of the subjects started with a block where they had to respond to the local level; the other half started with a global block. The subjects switched between the two target levels 
across blocks. Together, the four different blocks were run 10 times, distributed over about five sessions. Therefore, again, we obtained 80 responses for each experimental condition per subject.

Eight right-handed (by self-report) subjects ( 4 male and 4 female), who ranged in age from 20 to 33 years and who had normal or corrected-to-normal visual acuity, participated in the experiment.

\section{Latencies}

\section{Results and Discussion}

With the data of Experiment 2 (see Figure 4), a fourfactor ANOVA for repeated measurements on all factors was computed. A reliable effect was produced by the position factor $[F(2,14)=15.8, p<.001]$; this was due to the faster responses obtained to the stimuli presented at the CVF (LVF, $556 \mathrm{msec}$; CVF, $545 \mathrm{msec}$; RVF, $552 \mathrm{msec}$ ). Also, the factor of stimulus type was significant $[F(2,14)=$ $47.6, p<.001]$. Highpass filtering again led to consider- ably slowed responses ( $602 \mathrm{msec}$ ), as compared with those for the filled $(521 \mathrm{msec})$ and outlined $(530 \mathrm{msec})$ stimuli. However, the latencies for the filled and outlined stimuli also differed significantly $[F(1,7)=25.0, p<$ $.01]$. Furthermore, the consistency effect was significant [consistent, $545 \mathrm{msec}$; inconsistent, $557 \mathrm{msec} ; F(1,7)=$ $24.4, p<.01]$, while the target-level factor only just failed $[F(1,7)=5.56, p<.06]$.

There was, however, a two-way interaction between position and target level $[F(2,14)=6.11, p<.05]$. If we analyze the data only for the LVF and RVF, it turns out that the responses to the local level were faster for stimuli presented at the RVF [556 msec vs. $568 \mathrm{msec} ; F(1,7)=6.22$, $p<.05]$; there was no difference for the global level (LVF, $544 \mathrm{msec}$; RVF, $546 \mathrm{msec}$ ). Thus, there were no hemispheric differences for the responses to the global target level. There was still, however, a visual-field ef-

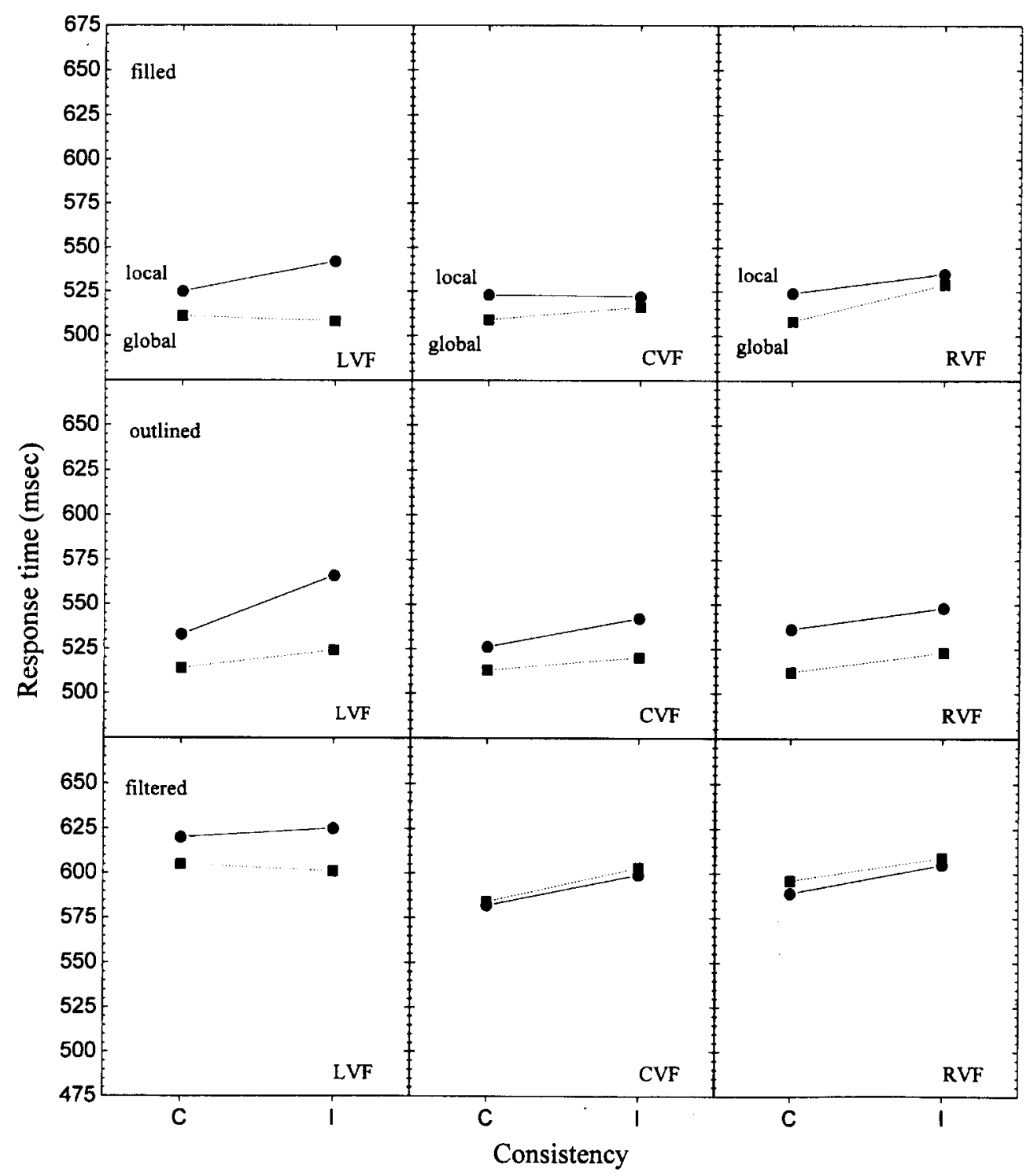

Figure 4. Results of Experiment 2. 
Table 2

Error Rates of Experiment 2 in Percentages

\begin{tabular}{|c|c|c|c|c|c|c|c|}
\hline \multirow[b]{2}{*}{ Stimulus } & \multirow{2}{*}{$\begin{array}{l}\text { Target } \\
\text { Level }\end{array}$} & \multicolumn{2}{|c|}{ LVF } & \multicolumn{2}{|c|}{$\mathrm{CVF}$} & \multicolumn{2}{|c|}{ RVF } \\
\hline & & Con. & Incon. & Con. & Incon. & Con. & Incon. \\
\hline \multirow[t]{2}{*}{ Filled } & global & 2.4 & 3.6 & 1.6 & 4.5 & 1.5 & 4.9 \\
\hline & local & 2.0 & 3.4 & 3.3 & 3.8 & 3.7 & 4.9 \\
\hline \multirow[t]{2}{*}{ Outlined } & global & 2.1 & 3.1 & 2.2 & 5.6 & 1.6 & 4.6 \\
\hline & local & 2.0 & 5.6 & 3.4 & 4.8 & 3.3 & 4.0 \\
\hline \multirow[t]{2}{*}{ Filtered } & global & 5.9 & 5.0 & 5.2 & 5.6 & 6.0 & 7.5 \\
\hline & local & 7.9 & 7.5 & 4.3 & 5.1 & 4.8 & 6.3 \\
\hline
\end{tabular}

Note- $-\mathrm{LVF}=$ left visual field, $\mathrm{CVF}=$ center visual field, $\mathrm{RVF}=$ right visual field. Con. $=$ consistent, Incon. $=$ inconsistent.

fect for the local level, although it was considerably reduced compared with that in Experiment 1.

Furthermore, there was an interaction between stimulus type and target level $[F(2,14)=7.20, p<.01]$. This was due to the fact that there was no difference between responses to the global $(600 \mathrm{msec})$ and local (603 $\mathrm{msec})$ levels of the filtered stimuli. Thus, in this experiment, filtering had the effect of eliminating the global advantage, at least when the data were averaged across positions.

Of the three-way interactions, the one between position, stimulus type, and consistency $[F(4,28)=3.44, p<$ $.05]$ was significant. This was due to an interaction between stimulus type and consistency for the LVF data $[F(2,14)=5.07, p<.05]$, which was absent in the RVF data. A further analysis revealed that only the outlined stimuli produced a significant LVF consistency effect $[F(1,7)=28.1, p<.01]$.

In addition, the interaction between position, target level, and consistency $[F(2,14)=6.73, p<.01]$ was significant. This was due to a target-level $\times$ consistency interaction in the LVF data $[F(1,7)=6.23, p<.05]$, which was absent in the RVF data. The two-way interaction in the LVF data indicates that a reliable consistency effect was present only for the local target level $[F(1,7)=14.4$, $p<.01]$.

\section{Error Rates}

In this experiment, the errors were even fewer $(4.2 \%)$ than those made in the first experiment (see Table 2). Again, the arcsin transformed error rates were subjected to an ANOVA, and the stimulus-type factor was significant $[F(2,14)=4.73, p<.05]$. More errors occurred for the filtered stimuli than for the other stimuli (filled, $3.3 \%$; outlined, $3.5 \%$; filtered, $5.9 \%$ ). Also significant was the consistency factor [consistent, $3.5 \%$; inconsistent, $5.0 \% ; F(1,7)=7.57, p<.05]$. Finally, there was a reliable stimulus type $\times$ consistency interaction $[F(2,14)$ $=4.37, p<.05]$. This was due to the fact that the filtered stimuli produced only a small consistency effect$0.5 \%$-as compared with $1.78 \%$ for the filled and $2.2 \%$ for the filtered stimuli. Thus, in this experiment, too, there was no indication of any speed-accuracy tradeoff effects.

\section{COMPARISON BETWEEN EXPERIMENTS 1 AND 2}

For a statistical comparison of the two experiments, all data were subjected to a five-factor ANOVA with the four within-subjects factors, which are identical for the two experiments, and the additional between-subjects factor of task mode (blocked target level, randomized target level). As can be seen by comparing Figures 3 and 4 , the responses in Experiment 2 were generally faster than those in Experiment $1[F(1,14)=6.54, p<.05]$. On average, blocking the target level speeded the responses up by about $100 \mathrm{msec}$.

There was also a significant two-way interaction between task mode and consistency $[F(1,14)=8.97, p<$ $.05]$. The consistency effect was larger in the randomized condition $(36 \mathrm{msec})$ than in the blocked condition $(12 \mathrm{msec})$. Also, there was a three-way interaction between task mode, position, and target level $[F(1,14)=$ $10.1, p<.01]$. While the latency differences between the responses to the global and local level were $63 \mathrm{msec}$ for the LVF and $10 \mathrm{msec}$ for the RVF stimuli in the first experiment, they were $24 \mathrm{msec}$ for the LVF and $10 \mathrm{msec}$ for the RVF stimuli in the second experiment. Thus, the global response time advantage for the stimuli presented at the LVF was considerably reduced by blocking the target level. Finally, there was also a significant five-way interaction. This is not surprising in consideration of the fourway interaction in Experiment 1, which was absent in the second experiment.

\section{GENERAL DISCUSSION}

The experiments were carried out to explore the role of low spatial frequencies for the global-advantage phenomenon. A further goal was to investigate whether the hemispheres were specialized for processing global or local information and/or for certain spatial frequencies.

Surprisingly, in the first experiment, where the target level was randomized, elimination of low spatial frequencies had no effect other than to generally slow down the responses, that is, the global response time advantage and the interference pattern were not affected. This demonstrates that they are neither necessary for a global response time advantage or for global-on-local interference. Rather, the results suggest that global advantage is largely an attentional phenomenon. Interestingly, there was interference not only from global to local, but also from local to global, although, on average, the responses to the global level were faster. This result is incompatible with Navon's (1977) global-precedence hypothesis, and suggests that both target levels are processed in parallel.

In considering the data for the filtered stimuli, the question arises as to how the global patterns are processed in the absence of low spatial frequencies. If the information is transmitted only by high-spatial-frequency channels, then the global form might be encoded by some 
kind of hierarchical feature processing (e.g., Biederman, 1987; Rumelhart, 1970). However, in this case, one would expect the responses to the local level to be faster than those to the global level. An alternative hypothesis, which has been suggested by Badcock et al. (1990), is to assume that high-spatial-frequency channels provide local contrast estimates on which grouping processes can operate simultaneously at different scales. To explain the present results, one would have to presume that global information was extracted at a higher rate than local information from the filtered stimuli.

While the expected filtering effects did not occur in Experiment 1, there appeared to be appreciable hemispheric differences. The responses to the global level were faster for stimuli presented at the LVF than for those presented at the RVF, and the opposite held true for responses to the local level. Since global responses were generally faster in most conditions, a substantial global response time advantage occurred only for stimuli presented at the LVF. Interestingly, no hemispheric differences could be observed with respect to the presence or absence of low spatial frequencies. This indicates that the hemispheres are not differentially sensitive to low spatial frequencies. Unexpectedly, however, the outlined stimuli, which have additional energy at their high spatial frequencies, produced a large amount of local-on-global interference when presented at the RVF. Further research will show whether this effect is reproducible.

In Experiment 2, a constant target level generally decreased the response times and error rates in comparison to the first experiment. Furthermore, the interference is reduced under this condition. These performance improvements were expected in view of the sequential effects found in Experiment 1, which resemble those observed by Ward (1982). If one considers Ward's and the present results, on the one hand, and the observed effects in taskswitching experiments (e.g., Allport et al., 1994; Rogers \& Monsell, 1995; Strayer \& Kramer, 1994), on the other, then it is surprising how similar, despite the rather different tasks involved, they are. This suggests that the postulated processes of focusing on, or allocating attention to, the global or local level, or Ward's level readiness, are special cases of more general cognitive control processes. For instance, "adjusting the attentional spotlight to an appropriate size" in the present context, could also be expressed more generally as "configuring the supervisory attentional system" (e.g., Allport et al., 1994).

In any case, the performance differences between the present two experiments can be well accounted for by taskset mechanisms. If, as in Experiment 1, frequent switching between the target levels is required, then the taskrelevant cognitive system has to be reconfigured each time. Allport et al. (1994) speculate that task switching consists of disengagement from the readiness for one task and preparation for the other. After Rogers and Monsell (1995), one could assume that, in the present case, both the global and local letters evoked a tendency to respond according to stimulus-response mapping, and that the intention to respond to the letter at the nontarget level had to be overridden in order to avoid a possible response conflict. Anyway, such a reconfiguration or disengagement process produces considerable costs in response times and error rates, particularly since it can be achieved only partly by endogenous (top-down) control. Consequently, a residual switching cost is independent of the responsestimulus interval. For completing a task switch, exogenous (bottom-up) control is necessary, that is, the arrival of an appropriate stimulus (cf. Allport et al., 1994; Rogers \& Monsell, 1995). Also, the latter fact produces a large amount of crosstalk between the tasks or target levels, which can explain the increased interference in Experiment 1 as compared with Experiment 2.

The constant target level in Experiment 2 also had the effect that, on average, there were no latency differences between the responses to the global and local levels for the filtered stimuli. This can be seen in Figure 5, where the averaged latencies for responding to the global and local levels for the three stimulus types in the two experiments are depicted. If only the data of the second experiment were collected and then averaged across the different positions, it would have been considered as support for the hypothesis that low spatial frequencies were responsible for the global advantage. However, as the data of the two experiments demonstrate, such an account is too simple.

The results of the present experiments suggest that the global advantage is neither a purely sensory nor a purely attentional effect. Rather, sensory as well as attentional mechanisms seem to be involved. This view agrees with that of Lamb and his coworkers (e.g., Lamb \& Yund, 1993; Robertson \& Lamb, 1991). They also came to the conclusion that sensory as well as higher order mechanisms played a role in the processing of compound stimuli, where, however, the latter are seen as the more important.

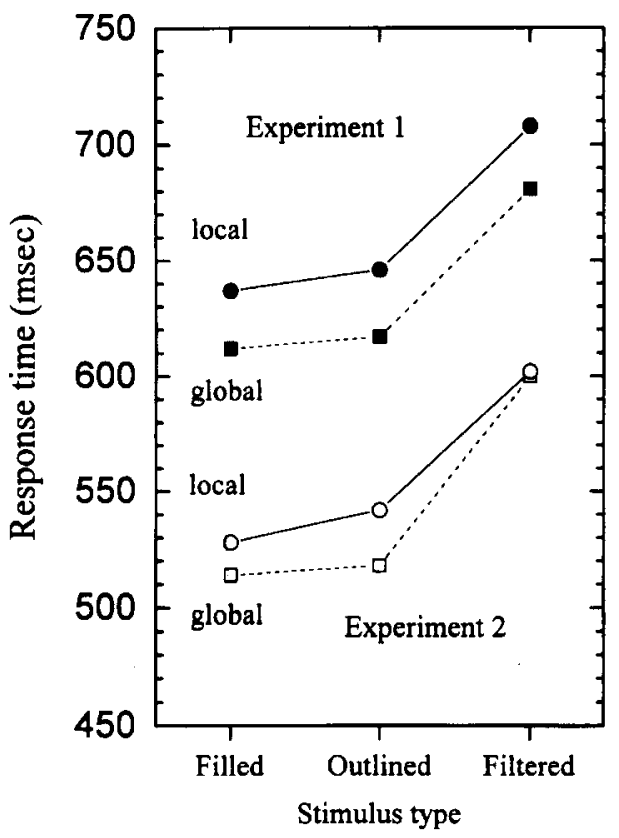

Figure 5. Mean results of global and local processing for the three stimulus types in both experiments. 
The present data indicate that, if fast processing of the information at a certain level is not possible, say because frequent level switching prevents an optimal configuration of the supervisory system, the contribution of low spatial frequencies for the global advantage decreases. Whether the effects of additional high spatial frequencies increase in this case, as the data for the outlined stimuli suggest, would be an interesting question for further research. In any case, the contribution of the characteristic differences between the attentional channels (in the sense of Norman, 1969) and control mechanisms for processing local and global information obviously increase. To explain the attentional basis for the global advantage, one can, for instance, adopt Miller's (1981) two-factor model, which states that the global channel is stronger than the local channel, and that the strength of the two channels cannot be manipulated independently. However, as the present results suggest, one should also consider task-set control processes and their differences with respect to local and global information.

Except for the peculiar effects of the outlined stimuli, neither of the reported experiments provides evidence that the hemispheres are differentially sensitive to certain spatial frequencies. In any case, the hypothesis that the right hemisphere can be regarded as an early processor especially qualified for processing the output of lowspatial-frequency channels (Sergent, 1982) is not confirmed. Rather, the data support the hypothesis that the hemispheres are specialized for processing either local or global information, which, however, is different from processing high or low spatial frequencies, respectively. Particularly appreciable are the hemispheric differences in Experiment 1 . They are in line with the hypothesis that while the right hemisphere is specialized for processing global information, the left hemisphere is superior at processing local information.

An interesting question concerns whether both hemispheres, despite their specializations, process both global and local information. If so, to what extent do the hemispheres interact during their processing of compound stimuli? Zaidel and Rayman (1994) provide some theoretical data patterns, which would be expected for different models of interhemispheric interaction. To a large extent, their method is based on the comparison of performance for redundant bilateral presentations - that is, presentations in which a copy of the stimulus is presented to the LVF and RVF simultaneously - with that for lateral presentations. Although it could be questioned whether such a comparison made sense, it might nevertheless be useful in approaching a first answer regarding interhemispheric communication. If, for instance, the performance of stimuli presented at one VF is similar to that of stimuli presented bilaterally, and the performance of the stimuli presented at the other VF is worse, then this pattern is compatible with both the "direct-access" and the "callosal-relay" models. The direct-access model assumes that each hemisphere is capable of performing the task, but that they may use differently efficient processing strategies. Information is processed without recourse of interhemispheric exchange. On the other hand, the callosal-relay model states that the task is performed exclusively in one hemisphere, and that the information must be relayed to the other hemisphere when it is presented at the inappropriate VF. Zaidel and Rayman (1994) consider further the "interhemispheric-interaction" model, which assumes that each hemisphere contributes to the task, and that there is an additional interhemispheric exchange. For the present case, one could consider that the CVF stimuli make some kind of redundant bilateral presentations. It seems not unreasonable to assume that a sensory copy of the CVF stimuli is transferred at an equal rate to each hemisphere.

If we examine the latency data of Experiment 1 within this framework, it is obvious that the global CVF data are more similar to the LVF data than to the RVF data. On the other hand, the local CVF data show greater resemblance to the RVF data. As mentioned above, this pattern is compatible with both the direct-access model and the callosal-relay model. The latter model would assume that local information was processed exclusively in the $\mathrm{LH}$ and that global information was processed exclusively in the RH. However, if we also take the data of Experiment 2 into account, then the callosal-relay model can be excluded, since no latency difference occurred between the hemispheres at the global level. This is incompatible with the callosal-relay model, which predicts a time difference that is at least as large as the transmission time between the hemispheres.

Thus, the data seem to be in line with the directaccess model. However, the pattern becomes more complicated if we also take the interference effects into account. Obviously, there is a dissociation of response time advantage and interference. For instance, although the latency difference between the responses to global and local is larger for the filled LVF stimuli than for the filled RVF stimuli in Experiment 1, the interference is similar for LVF and RVF stimuli. A striking dissociation is also obvious for the outlined stimuli. The dissociation is in line with Lamb and Robertson's (1989) results, and indicates that response time advantage and interference are caused by different processes. This hypothesis is also supported by neuropsychological results (e.g., Lamb, Robertson, \& Knight, 1989), where interference was eliminated after brain lesioning, which prevented communication between the hemispheres (cf. Humphreys et al., 1985; Robertson et al., 1993). Robertson and Lamb (1991) speculate that interference is mediated by the posterior corpus callosum.

In consideration of the dissociation of response time advantage and interference, the present data should be interpreted in the context of the interhemispheric-interaction model. Both hemispheres are capable of processing both global and local information, although with differential efficiency. It can further be assumed that both hemispheres have access to all the information, even during lateralized presentation, and that the $\mathrm{RH}$ dominates global processing by interhemispheric control mechanisms, while the LH dominates local processing (cf. Zaidel 
\& Rayman, 1994). Such an account would also be compatible with the task-set explanation of the reduced interference in Experiment 2. However, it was not only the interference that was reduced by the constant target level; the visual-field differences, especially the global response time advantage for the LVF stimuli, were too. This suggests that a constant global or local target level allows the hemispheres to optimize, according to their specializations, their control mechanisms for dominating the respective information processing and to prevent crosstalk from the task-irrelevant processes that are dominated by the other hemisphere.

Finally, it bears mentioning that the reduced hemispheric differences in Experiment 2 could also explain why several other experiments failed to show functional asymmetries. Almost all of those studies, in which no significant or unexpected hemispheric differences were found, held the target level constant in each experimental block (Alivisatos \& Wilding, 1982; Boles, 1984; Boles \& Karner, 1996; Van Kleeck, 1989). One exception is Robertson et al. (1993), who obtained significant hemispheric effects with constant target levels. However, their effects were as small as those observed in the present Experiment 2. On the other hand, at least one (Sergent, 1982) of the two successful studies (Martin, 1979; Sergent, 1982) required divided attention. Dividing attention across target levels might be different from switching the focus of attention between the levels. However, it can be assumed that both tasks prevent the subjects from optimally focusing on a certain level. This probably leads to a relatively large amount of crosstalk between the mechanisms that are responsible for local or global processing. Such crosstalk seems to be important for producing significant hemispheric differences.

\section{REFERENCES}

Alivisatos, B., \& Wilding, J. (1982). Hemispheric differences in matching Stroop-type letter stimuli. Cortex, 18, 5-22.

Allport, A., Strles, E. A., \& Hsieh, S. (1994). Shifting intentional set: Exploring the dynamic control of tasks. In C. Umiltà \& M. Moscovitch (Eds.), Attention and performance XV (pp. 421-452). Cambridge, MA: MIT Press.

Badcock, J. C., Whitworth, F. A., Badcock, D. R., \& Lovegrove, W. J. (1990). Low-frequency filtering and the processing of localglobal stimuli. Perception, 19, 617-629.

BIEDERMAN, I. (1987). Recognition-by-components: A theory of human image understanding. Psychological Review, 94, 115-147.

BoER, L. C., \& Keuss, P. J. G. (1982). Global precedence as a postperceptual effect: An analysis of speed-accuracy tradeoff functions. Perception \& Psychophysics, 31, 358-366.

BoLEs, D. B. (1984). Global versus local processing: Is there a hemispheric dichotomy? Neuropsychologia, 22, 445-455.

Boles, D. B., \& Karner, T. A. (1996). Hemispheric differences in global versus local processing. Brain \& Cognition, 30, 232-243.

Bradshaw, J. L., \& Nettleton, N. C. (1981). The nature of hemispheric specialization in man. Behavioral \& Brain Sciences, 4, 51-63.

BREITMEYER, B. G. (1975). Simple reaction time as a measure of the temporal response properties of transient and sustained channels. $\mathrm{Vi}$ sion Research, 15, 1411-1412.

BREITMEYER, B. G. (1984). Visual masking. New York: Oxford University Press.

BRetTMEyeR, B. G, \& GANZ, L. (1977). Temporal studies with flashed gratings: Inferences about human transient and sustained channels.
Vision Research, 17, 861-865.

Campbell, F. W., \& Robson, J. G. (1968). Application of Fourier analysis to the visibility of gratings. Journal of Physiology, 197, 551-566.

DeValoIs, R. L., \& DeValors, K. K. (1988). Spatial vision. Oxford: Oxford University Press.

ERiksen, C. W., \& St. JAMES, J. D. (1986). Visual attention within and around the field of focal attention: A zoom lens model. Perception \& Psychophysics, 40, 225-240.

Felipe, A., Buades, M. J., \& Artigas, J. M. (1993). Influence of the contrast sensitivity function on the reaction time. Vision Research, 33, 2461-2466.

Graham, N. (1989). Visual pattern analyzers. New York: Oxford University Press.

Hoffman, J. E. (1980). Interaction between global and local levels of a form. Journal of Experimental Psychology: Human Perception \& Performance, 6, 222-234.

HÜBNER, R. (1993). Different ways of modeling spatial-frequency uncertainty in visual signal detection. Biological Cybernetics, 69 , 457-462.

HÜBNER, R. (1996). The efficiency of different cue types for reducing spatial-frequency uncertainty. Vision Research, 36, 401-408.

Hughes, H. C. (1986). Asymmetric interference between components of suprathreshold compound gratings. Perception \& Psychophysics, 40, 241-250.

Hughes, H. C., Fendrich, R., \& Reuter-Lorenz, P. A. (1990). Global versus local processing in the absence of low spatial frequencies. Journal of Cognitive Neuroscience, 2, 272-282.

Humphreys, B. W., Riddoch, M. J., \& Quinlan, P. T. (1985). Interactive processes in perceptual organization: Evidence from visual agnosia. In M. I. Posner \& O. S. M. Marin (Eds.), Attention \& performance XI (pp. 301-318). Hillsdale, NJ: Erlbaum.

JoNides, J., \& YANTIS, S. (1988). Uniqueness of abrupt visual onset in capturing attention. Perception \& Psychophysics, 43, 346-354.

Julesz, B., \& Papathomas, T. V. (1984). On spatial-frequency channels and attention. Perception \& Psychophysics, 36, 398-399.

KINCHLA, R. A. (1974). Detecting target elements in multielement arrays: A confusability model. Perception \& Psychophysics, 15, 149-158.

Kinchla, R. A., Solis-Macias, V., \& Hoffman, J. (1983). Attending to different levels of structure in a visual image. Perception \& Psychophysics, 33, 1-10

KINCHLA, R. A., \& WolfE, J. M. (1979). The order of visual processing: "Top-down," "bottom-up," or "middle-out." Perception \& Psychophysics, 25, 225-231.

Kitterle, F. L., Christman, S., \& Conesa, J. (1993). Hemispheric differences in the interference among components of compound gratings. Perception \& Psychophysics, 54, 785-793.

LAGASSE, L. L. (1993). Effects of good form and spatial frequency on global precedence. Perception \& Psychophysics, 53, 89-105.

LAMB, M. R., \& RoBERTSON, L. C. (1988). The processing of hierarchical stimuli: Effects of retinal locus, locational uncertainty, and stimulus identity. Perception \& Psychophysics, 44, 172-181.

LAMB, M. R., \& Robertson, L. C. (1989). Do response time advantage and interference reflect the order of processing of global- and locallevel information? Perception \& Psychophysics, 46, 254-258.

LAmb, M. R., Robertson, L. C., \& KNight, R. T. (1989). Attention and interference in the processing of global and local information: Effects of unilateral temporal-parietal junction lesions. Neuropsychologia, 27, 471-483.

LAMB, M. R., \& YUND, E. W. (1993). The role of spatial frequency in the processing of hierarchically organized stimuli. Perception \& Psychophysics, 54, 773-784.

Lovegrove, W. J., Lehmkuile, S., Baro, J. A., \& Garzia, R. (1991). The effects of uniform field flicker and blurring on the global precedence effect. Bulletin of the Psychonomic Society, 29, 289-291.

LupP, U., Hauske, G., \& Wolf, W. (1976). Perceptual latencies to sinusoidal gratings. Vision Research, 16, 969-972.

MARTIN, M. (1979). Local and global processing: The role of sparsity. Memory \& Cognition, 7, 476-484.

MiLLER, J. (1981). Global precedence in attention and decision. Journal of Experimental Psychology: Human Perception \& Performance, 7, 1161-1174

Navon, D. (1977). Forest before trees: The precedence of global fea- 
tures in visual perception. Cognitive Psychology, 9, 353-383.

Norman, D. A. (1969). Memory and attention. New York: Wiley.

Peterzell, D. H., Harvey, L. O., JR., \& Hardyck, C. D. (1989). Spatial frequencies and the cerebral hemispheres: Contrast sensitivity, visible persistence, and letter classification. Perception \& Psychophysics, 46, 443-455.

Pomerantz, J. R. (1983). Global and iocal precedence: Selective attention in form and motion perception. Journal of Experimental Psychology: General, 112, 516-540.

POSNER, M. I. (1980). Orienting of attention. Quarterly Journal of Experimental Psychology, 32, 3-25.

Roberrson, L. C., \& LAMB, M. R. (1991). Neuropsychological contributions to theories of part/whole organization. Cognitive Psychology, 23, 299-330.

RoBerTson, L. C., LAMB, M. R., \& ZAIDEL, E. (1993). Interhemispheric relations in processing hierarchical patterns: Evidence from normal and commisurotomized subjects. Neuropsychology, 7, 325-342.

ROGERS, R. D., \& MONSELl, S. (1995). Costs of a predictable switch between simple cognitive tasks. Journal of Experimental Psychology: General, 124, 207-231.

RumelhaRT, D. E. (1970). A multicomponent theory of the perception of briefly exposed visual displays. Journal of Mathematical Psychology, 7, 191-218.

SERGENT, J. (1982). The cerebral balance of power: Confrontation or cooperation. Journal of Experimental Psychology: Human Perception \& Performance, 8, 253-272.

SERGENT, J. (1987). Failures to confirm the spatial-frequency hypothesis: Fatal blow or healthy complication? Canadian Journal of Psychology, 41, 412-428.

Shulman, G. L., Sullivan, M. A., Gish, K., \& Sakoda, W. J. (1986). The role of spatial-frequency channels in the perception of local and global structure. Perception, 15, 259-273.

Stoffer, T. (1994). Attentional zooming and the global-dominance phenomenon: Effects of level-specific cueing and abrupt visual onset. Psychological Research, 56, 83-98.

Strayer, D. L., \& Kramer, A. F. (1994). Strategies and automaticity: II. Dynamic aspects of strategy adjustment. Journal of Experimental Psychology: Learning. Memory, \& Cognition, 20, 342-365.

ToDD, S., \& Kramer, A. F. (1994). Attentional misguidance in visual search. Perception \& Psychophysics, 56, 198-210.

TODD, S., \& VAN GELDER, P. (1979). Implications of a sustained-transient dichotomy for the measurement of human performance. Journal of Experimental Psychology: Human Perception \& Performance, $\mathbf{5}$, 625-638.

Tolmurst, D. J. (1975). Reaction times in the detection of gratings by human observers: A probabilistic mechanism. Vision Research, 15, 1143-1149.

VAN KLEECK, M. H. (1989). Hemispheric differences in global versus local processing of hierarchical visual stimuli by normal subjects: New data and a meta-analysis of previous studies. Neuropsychologia, 27, $1165-1178$.

WARD, L. M. (1982). Determinants of attention to local and global fea- tures of visual forms. Journal of Experimental Psychology: Human Perception \& Performance, 8, 562-581.

YANTIS, S., \& HillsTrom, A. (1994). Stimulus-driven attentional capture: Evidence from equiluminance visual objects. Journal of Experimental Psychology: Human Perception \& Performance, 20, 95-107.

YANTIS, S., \& JoNIDES, J. (1984). Abrupt visual onsets and selective attention: Evidence from visual search. Journal of Experimental Psychology: Human Perception \& Performance, 10, 601-621.

ZAIDEL, E., \& RAYMAN, J. (1994), Interhemispheric control in the normal brain: Evidence from redundant bilateral presentations. In C. Umiltà \& M. Moscovitch (Eds.), Attention and performance XV (pp. 476504). Cambridge, MA: MIT Press.

\section{NOTES}

1. One reviewer argued that the present experiment differed from others also with respect to the complex stimulus-response mapping. To look for a possible effect, the data for the consistent conditions (LVF and RVF data only) were analyzed again, but separately for form-compatible stimuli (same letters on both levels) and form-incompatible stimuli (different letters on both levels). It turned out that the responses to form-compatible stimuli were $30 \mathrm{msec}$ faster $[F(1,7)=10.7, p<.05]$ than those to the form-incompatible stimuli. There is further a four-way interaction between form compatibility (compatible, incompatible), position (LVF, RVF), and stimulus type and target level $[F(1,7)=5.75$, $p<.05]$. A detailed analysis revealed that this interaction was due to the outlined stimuli: The interaction between target level and position does not depend on form compatibility for the filled and filtered stimuli. The responses to the global level were faster for LVF stimuli than for RVF stimuli, and the opposite was true for responses to the local level. While the same pattern of results occurred for the form-incompatible outlined stimuli, it was reversed for the form-compatible outlined stimuli. Responses to the global level were faster to RVF stimuli than to LVF stimuli, and the opposite held for responses to the local level. This result is quite peculiar. Most important, however, there was no specific formcompatibility effect for the filtered stimuli.

2. For the present experiment, one might also ask whether there are sequential dependencies with respect to stimulus type. If the subjects concentrated on the characteristic spatial-frequency content of a certain stimulus type, then they could have had an advantage when the same stimulus type occurred in the subsequent trial. However, by computing a two-factor (stimulus type $X$ stimulus type in the preceding trial) ANOVA, the stimulus-type factor turned out to be significant $[F(2,14)=59.5$, $p<.001]$, which is not surprising. However, there was no reliable interaction $[F(4,28)=1.25, p>.30]$.

(Manuscript received June 26, 1995; revision accepted for publication March 17, 1996.) 